\section{International Scientific Journal Theoretical \& Applied Science}

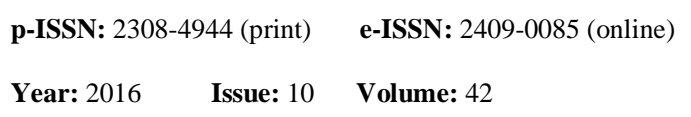

Published: $30.10 .2016 \quad$ http://T-Science.org

SECTION 1. Theoretical research in mathematics.
Nurlan Abiev candidate of physico-mathematical Sciences, associate Professor, chief of the Department of «Mathematics», Taraz State University named after M.Kh. Dulaty abievn@mail.ru

\section{Erik Bayandiev} researcher

Department of «Mathematics», Taraz State University named after M.Kh. Dulaty

Zhanar Keulimzhaeva researcher Department of «Mathematics», Eurasian National University named after L.N. Gumilyev

Alexandr Shevtsov candidate of technical sciences, Department of «Mathematics», Deputy Director on Science of faculty of information technologies, automation and telecommunications,

Taraz State University named after M.Kh. Dulaty Shev_AlexXXXX@mail.ru

\title{
INTERPRETATION OF PROPERTIES OF THE NORMALIZED RICCI FLOW ON THE WALLACH SPACES
}

\begin{abstract}
This papere is devoted to algorithm development and its implementation in Delphi language for the interpretation of qualitative picture of behavior of solutions of a system of differential equations obtained in the study of the evolution of Riemannian metrics on the Wallach spaces under the influence of the normalized Ricci flow. In the work a computer program with a user-friendly interface is developed. This program is directed to the visualization of the behavior of solutions of the system of differential equations with respect to the set of invariant Riemannian metrics admitting positive sectional curvature.

Key words: algorithm, the Wallach spaces, the normalized Ricci flow, Delphi.

Language: Russian

Citation: Abiev NA, Bayandiev EN, Keulimzhaeva ZA, Shevtsov AN (2016) INTERPRETATION OF PROPERTIES OF THE NORMALIZED RICCI FLOW ON THE WALLACH SPACES. ISJ Theoretical \& Applied Science, 10 (42): 58-65.

Soi: http://s-o-i.org/1.1/TAS-10-42-14 Doi: crossef http://dx.doi.org/10.15863/TAS.2016.10.42.14

\section{ИНТЕРПРЕТАЦИЯ СВОЙСТВ НОРМАЛИЗОВАННОГО ПОТОКА РИЧЧИ НА ПРОСТРАНСТВАХ УОЛЛАХА}

Аннотация: Настоящая статья посвящчена разработке алгоритма и его реализации на языке Delphi для интерпретации качественной картины поведения решений системы дифференциальных уравнений, полученной при изучении эволючии римановых метрик на пространствах Уоллаха под влиянием нормализованного потока Риччи. В работе разрабатьвается компьютерная программа, обладающая удобным пользовательским интерфейсом и направленная на визуализацию поведения решений системь дифференциальных уравнений относительно множества инвариантных римановых метрик, допускающих положительную секционную кривизну.

Ключевые слова: алгоритм, пространства Уоллаха, нормализованный поток Риччи, Delphi.
\end{abstract}

\section{Введение}

Настоящая статья посвящена разработке алгоритма и его реализации на языке Delphi для интерпретации качественной картины поведения решений следующей системы автономных дифференциальных уравнений

$$
\begin{aligned}
& x^{\prime}(t)=(x-1)(x-2 a x y-2 a y), \\
& y^{\prime}(t)=(y-1)(y-2 a x y-2 a x),
\end{aligned}
$$




\begin{tabular}{|c|c|c|c|c|c|c|}
\hline \multirow{4}{*}{ Impact Factor: } & ISRA (India) & $=1.344$ & SIS (USA) & $=0.912$ & ICV (Poland) & $=6.630$ \\
\hline & ISI (Dubai, UAF & $=0.829$ & РИНЦ (Russia) & $=0.234$ & PIF (India) & $=1.940$ \\
\hline & GIF (Australia) & $=0.564$ & ESJI (KZ) & $=1.042$ & IBI (India) & $=4.260$ \\
\hline & JIF & $=1.500$ & SJIF (Morocco) & $=2.031$ & & \\
\hline
\end{tabular}

где $a$-числовой параметр, принадлежащий интервалу $(0,1 / 2)$. Отметим, что система (1) получена в работе [1] при изучении эволюции римановых метрик на обобщенных пространствах Уоллаха под влиянием нормализованного потока Риччи (историю вопроса и необходимые определения читатель может найти в $[2,3]$ и цитируемой в них литературе). В данной работе предлагается компьютерная программа, обладающая удобным пользовательским интерфейсом и направленная на визуализацию результатов, полученных в работах $[1,4]$ о поведении решений системы (1) относительно области (см. ее описание в разделе 1) евклидовой плоскости, имеющей геометрический смысл множеств метрик положительной секционной кривизны.

\section{1. Предварительные сведения}

1.1. Уравнение нормализованного потока Риччи на обобщенных пространствах Уоллаха. Понятие обобщенного пространства Уоллаха введено в работах $[5,6,7]$. Согласно этим работам каждое обобщенное пространство Уоллаха описывается тройкой параметров $a_{i} \in(0,1 / 2]$. На обобщенных пространствах Уоллаха уравнение нормализованного потока Риччи может быть сведено к следующей системе нелинейных обыкновенных дифференциальных уравнений, полученной и изученной в [2]:

$$
\frac{d x_{1}}{d t}=f\left(x_{1}, x_{2}, x_{3}\right), \quad \frac{d x_{2}}{d t}=g\left(x_{1}, x_{2}, x_{3}\right), \quad \frac{d x_{3}}{d t}=h\left(x_{1}, x_{2}, x_{3}\right),
$$

где $x_{i}=x_{i}(t)>0$ - параметры инвариантной

римановой метрики, $i=1,2,3$,

$$
\begin{aligned}
& f\left(x_{1}, x_{2}, x_{3}\right)=-1-a_{1} x_{1}\left(\frac{x_{1}}{x_{2} x_{3}}-\frac{x_{2}}{x_{1} x_{3}}-\frac{x_{3}}{x_{1} x_{2}}\right)+x_{1} B \\
& g\left(x_{1}, x_{2}, x_{3}\right)=-1-a_{2} x_{2}\left(\frac{x_{2}}{x_{1} x_{3}}-\frac{x_{3}}{x_{1} x_{2}}-\frac{x_{1}}{x_{2} x_{3}}\right)+x_{2} B \\
& h\left(x_{1}, x_{2}, x_{3}\right)=-1-a_{3} x_{3}\left(\frac{x_{3}}{x_{1} x_{2}}-\frac{x_{1}}{x_{2} x_{3}}-\frac{x_{2}}{x_{1} x_{3}}\right)+x_{3} B, \\
& B:=\left(\frac{1}{a_{1} x_{1}}+\frac{1}{a_{2} x_{2}}+\frac{1}{a_{3} x_{3}}-\frac{x_{1}}{x_{2} x_{3}}-\frac{x_{2}}{x_{1} x_{3}}-\frac{x_{3}}{x_{1} x_{2}}\right)\left(\frac{1}{a_{1}}+\frac{1}{a_{2}}+\frac{1}{a_{3}}\right)^{-1}, \quad a_{i} \in(0,1 / 2] .
\end{aligned}
$$

\section{2. Вывод системы (1) $u$} классификационные результаты. Пусть $a_{1}=$ $a_{2}=a_{3}:=a$. Тогда, как показано в [1], введением инвариантных координат $x=\frac{x_{3}}{x_{1}}$, $y=\frac{x_{3}}{x_{2}}, \quad$ из системы (2) можно получить систему (1). Точку $\left(x_{0}, y_{0}\right)$, удовлетворяющую равенствам $P\left(x_{0}, y_{0}\right)=Q\left(x_{0}, y_{0}\right)=0$, называют особой точкой плоской системы $x^{\prime}=P(x, y), \quad y^{\prime}=Q(x, y)$. Пусть $\lambda_{1}, \lambda_{2}$ собственные значения матрицы Якоби

$$
J=J\left(x_{0}, y_{0}\right)=\left.\left(\begin{array}{ll}
P_{x} & P_{y} \\
Q_{x} & Q_{y}
\end{array}\right)\right|_{(x, y)=\left(x_{0}, y_{0}\right)} \text { и пусть }
$$

без ограничения общности $\left|\lambda_{1}\right| \leq\left|\lambda_{2}\right|$. Согласно теории особая точка плоской системы может иметь следующие типы (см., например, [8]): невырожденный тип $\left(\lambda_{1} \neq 0, \lambda_{2} \neq 0\right)$; полугиперболический тип $\left(\lambda_{1}=0, \quad \lambda_{2} \neq 0\right)$; нильпотентный тип $\left(\lambda_{1}=\lambda_{2}=0, \quad J \neq 0\right)$; линейно нулевой тип ( $J=0)$. Как следует из результатов [2,4], система (1) имеет единственную особую точку $(1,1)$ линейно нулевого типа, являющуюся седлом с шестью гиперболическими секторами. 


\begin{tabular}{l|lr|ll|ll} 
& ISRA (India) & $=\mathbf{1 . 3 4 4}$ & SIS (USA) & $=\mathbf{0 . 9 1 2}$ & ICV (Poland) & $=\mathbf{6 . 6 3 0}$ \\
Impact Factor: & ISI (Dubai, UAE) $=\mathbf{0 . 8 2 9}$ & PUHЦ (Russia) $=\mathbf{0 . 2 3 4}$ & PIF (India) & $=\mathbf{1 . 9 4 0}$ \\
& GIF (Australia) & $\mathbf{0 . 5 6 4}$ & ESJI (KZ) & $=\mathbf{1 . 0 4 2}$ & IBI (India) & $=\mathbf{4 . 2 6 0}$ \\
& JIF & $=\mathbf{1 . 5 0 0}$ & SJIF (Morocco) & $=\mathbf{2 . 0 3 1}$ & & \\
\hline
\end{tabular}

1.3. Множество D метрик положительной секиионной кривизныл. Множество D ограничивается кривыми, заданными следующими уравнениями (см. рисунок 1)

$$
\begin{aligned}
& x^{2} y^{2}-2 x^{2} y+2 x y^{2}+x^{2}+2 x y-3 y^{2}=0 \\
& x^{2} y^{2}+2 x^{2} y-2 x y^{2}-3 x^{2}+2 x y+y^{2}=0 \\
& 3 x^{2} y^{2}-2 x^{2} y-2 x y^{2}-x^{2}+2 x y-y^{2}=0
\end{aligned}
$$

Объектом нашего рассмотрения являются пространства Уоллаха

$$
S U(3) / T_{\max }, S p(3) / S p(1) \times S p(1) \times S p(1), \quad F_{4} / \operatorname{Spin}(8),
$$

которые являются также обобщенными пространствами Уоллаха, соответствующими значениям $a=\frac{1}{6}, a=\frac{1}{8}, a=\frac{1}{9}$.

\section{2. Разработка аппарата}

Воспользуемся одношаговым методом Рунге-Кутты (см. детали в работах $[9,10]$ ) для решения системы дифференциальных уравнений (1), представим ее в нормальной форме Коши, т.е. в следующей форме записи:

$$
\dot{x}(t)=f(x(t), t) ; \quad x\left(t_{0}\right)=x_{0}
$$

где $x$ - вектор решений; $f$ - вектор функций правых частей уравнений.

Для системы (3) вектор решений состоит из двух переменных, а вектор правых частей - из двух функций этих переменных и независимой переменной $t$, стоящих в правых частях уравнений. Если известно решение в некотором узле интегрирования $t_{k-1}$, то, применив, формулы Рунге-Кутты, можно выполнить один шаг интегрирования и вычислить приближенное решение в следующем узле $t_{k}=t_{k-1}+h$. Для выполнения шага потребуется несколько раз вычислить правые части уравнения (3) при различных значениях аргументов. Количество вычислений правых частей на шаге определяется порядком метода. Процедура шага интегрирования повторяется рекуррентно до тех пор, пока значение независимой переменной не достигнет правого конца отрезка интегрирования. [9]

Метод Рунге-Кутты четвертого порядка описывается следующими формулами:

$$
x\left(t_{k}\right)=x\left(t_{k-1}\right)+h / 6 *\left(F_{1}+2 *\left(F_{2}+F_{3}\right)+F_{4}\right)(4)
$$

где

$$
\left\{\begin{array}{l}
F_{1}=f\left(x\left(t_{k-1}\right), t_{k-1}\right) \\
F_{2}=f\left(x\left(t_{k-1}\right)+h / 2 * F_{1}, t_{k-1}+h / 2\right) \\
F_{3}=f\left(x\left(t_{k-1}\right)+h / 2 * F_{2}, t_{k-1}+h / 2\right) \\
F_{4}=f\left(x\left(t_{k-1}\right)+h * F_{3}, t_{k-1}+h\right)
\end{array}\right.
$$

Для вычисления правых частей по формуле (5) подпрограмма четыре раза обращается к специальной подпрограмме вычислений правых частей, которая составляется заново для каждого конкретного вида уравнения (3). Перед каждым таким обращением вычисляются аргументы правых частей для очередной формулы (5), а после этого добавляется очередное слагаемое в формулу (4). После последнего (четвертого) обращения с помощью формулы (5) пересчитывается значение вектора решений в новом узле интегрирования.

Приведем блок схему разработанной программы реализующей метод Рунге-Кутты четвертого порядка для системы (1): 


\begin{tabular}{|c|c|c|c|c|c|c|}
\hline Impact Factor: & $\begin{array}{l}\text { ISRA (India) } \\
\text { ISI (Dubai, UAE } \\
\text { GIF (Australia) } \\
\text { JIF }\end{array}$ & $\begin{array}{l}=1.344 \\
=0.829 \\
=0.564 \\
=1.500\end{array}$ & $\begin{array}{l}\text { SIS (USA) } \\
\text { PИНЦ (Russia) } \\
\text { ESJI (KZ) } \\
\text { SJIF (Morocco }\end{array}$ & $\begin{array}{l}=0.912 \\
=0.234 \\
=1.042 \\
=\mathbf{2 . 0 3 1}\end{array}$ & $\begin{array}{l}\text { ICV (Poland) } \\
\text { PIF (India) } \\
\text { IBI (India) }\end{array}$ & $\begin{array}{l}=6.630 \\
=1.940 \\
=4.260\end{array}$ \\
\hline
\end{tabular}

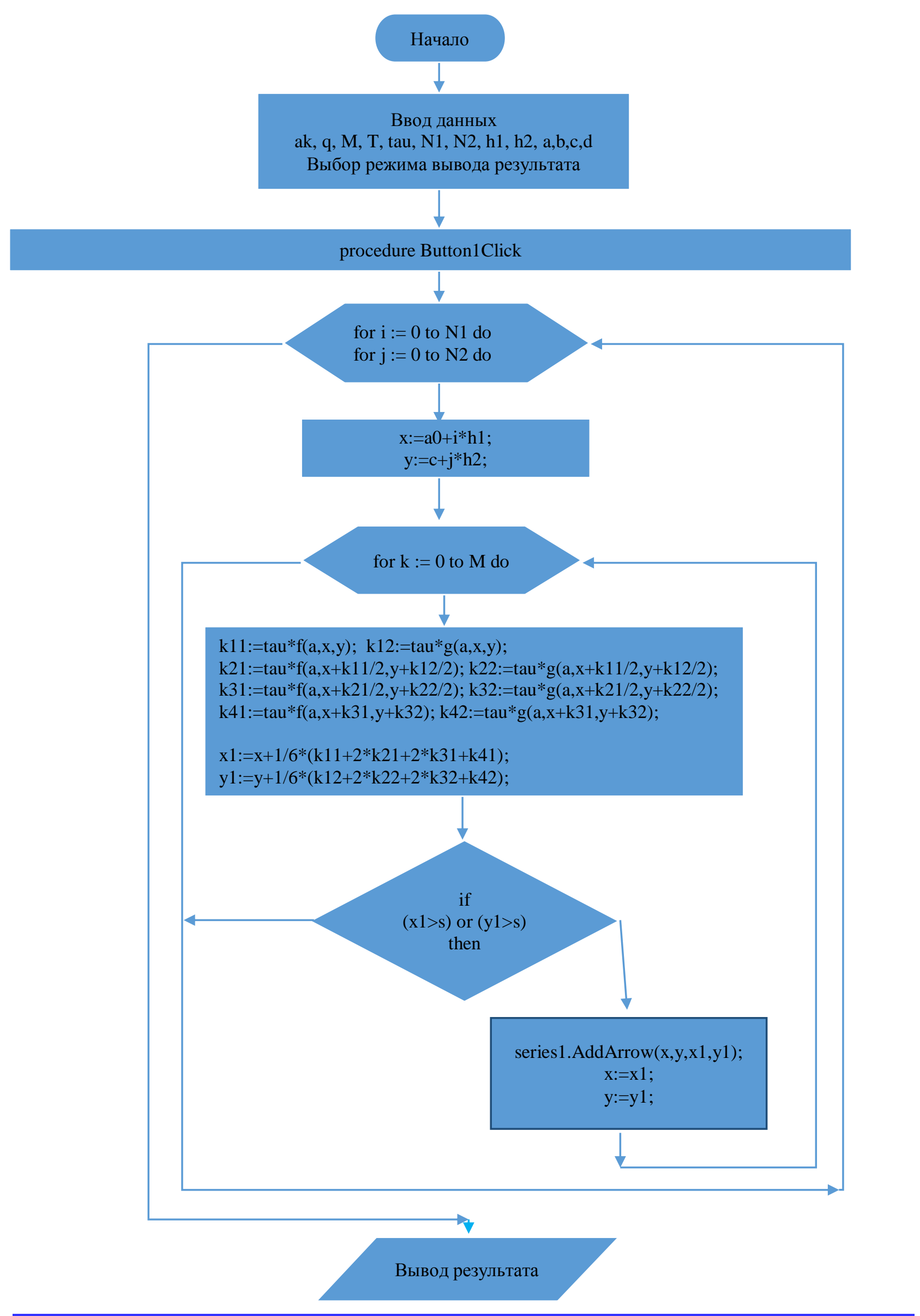

ISPC Computer technologies in science, 


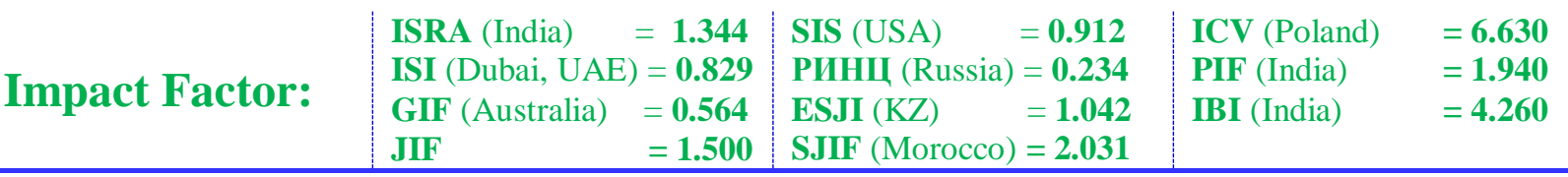

Функции $\mathrm{f}(\mathrm{a}, \mathrm{x}, \mathrm{y})$ и $\mathrm{g}(\mathrm{a}, \mathrm{x}, \mathrm{y})$ в данной блок схеме представляют собой подпрограммы:
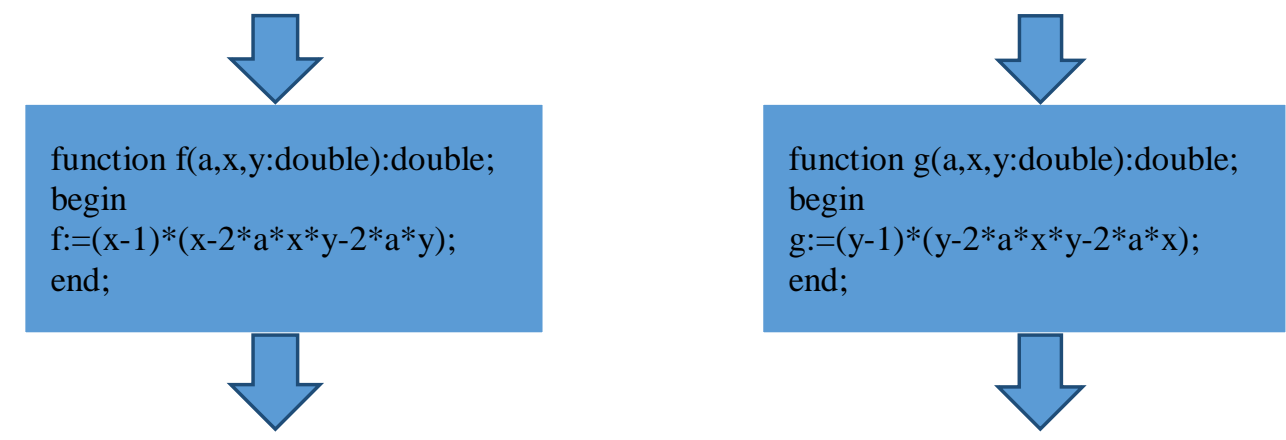

\section{3. Интерпретация результатов}

Шаг сетки выбирается автоматически по

А теперь испытаем работу созданной нами программы при значениях

$$
a=\frac{1}{6}, a=\frac{1}{8}, a=\frac{1}{9} .
$$

Для этого на левой панели вводим коэффициент $a$. Также имеется возможность выбора шага по х и по у, а также количество узлов расчетной сетки, и коэффициенты входящие в систему (1). введенным границам интервала и количеству узлов.

Получаем фазовые портреты, показанные на рисунке 2 для $\mathrm{a}=1 / 6$; для $\mathrm{a}=1 / 8$ результаты показаны на рисунке 3 ; для $a=1 / 9$ результаты показаны на рисунке 4. Преимущество программы состоит в том, что мы можем строить картину фазового потока системы (1) по отношению к области D при произвольных значениях параметра $a$, например, на рисунке 5 изображен случай при $a=\frac{1}{15}$.

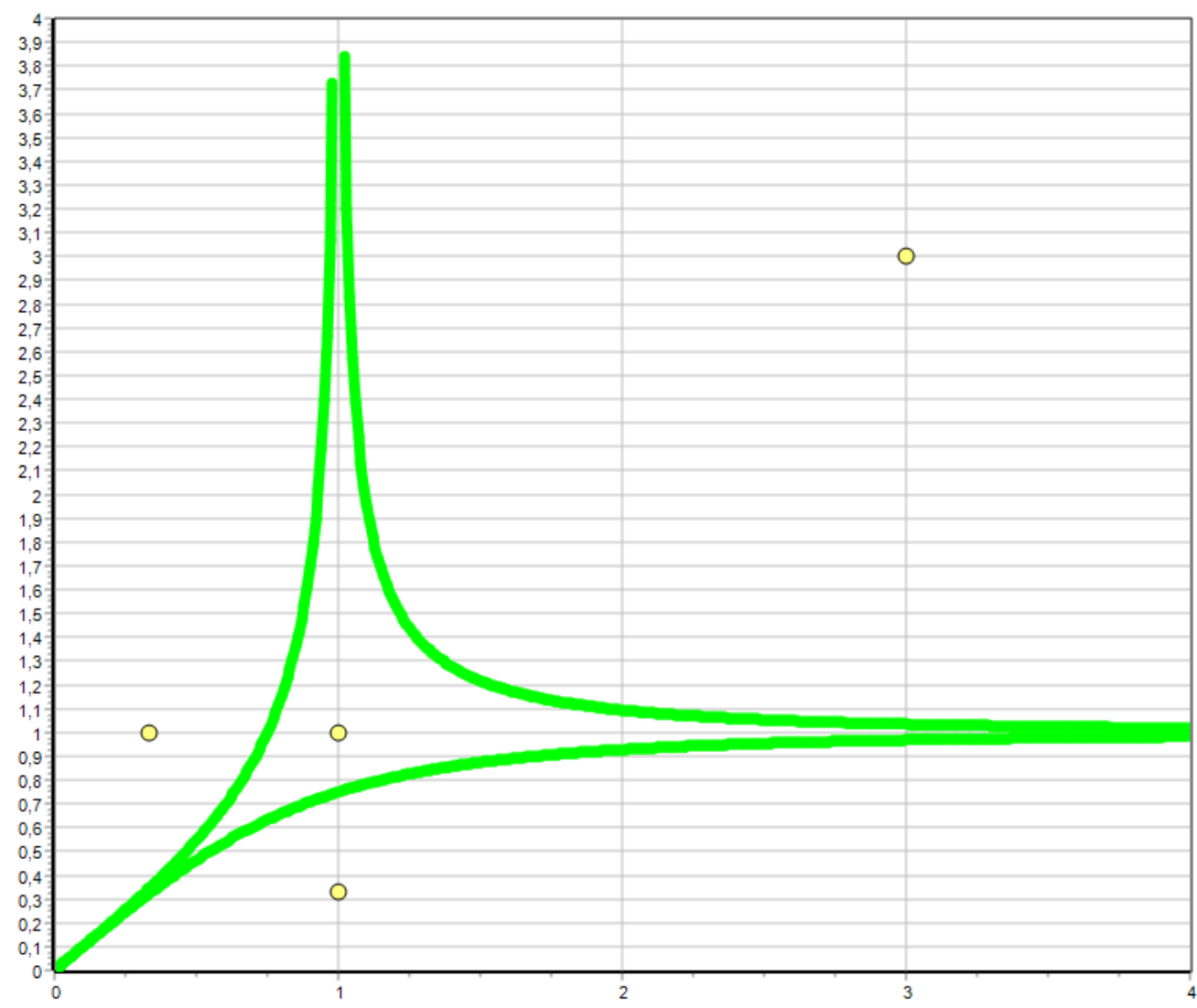

Рисунок 1 - Множество D и его граница.

ISPC Computer technologies in science, 


\begin{tabular}{|c|c|c|c|c|c|c|}
\hline Impact Factor: & $\begin{array}{l}\text { ISRA (India) } \\
\text { ISI (Dubai, UAE } \\
\text { GIF (Australia) } \\
\text { JIF }\end{array}$ & $\begin{array}{r}=1.344 \\
=0.829 \\
=0.564 \\
=1.500\end{array}$ & $\begin{array}{l}\text { SIS (USA) } \\
\text { PИНЦ (Russia) } \\
\text { ESJI (KZ) } \\
\text { SJIF (Morocco) }\end{array}$ & $\begin{array}{l}=0.912 \\
=0.234 \\
=1.042 \\
=2.031\end{array}$ & $\begin{array}{l}\text { ICV (Poland) } \\
\text { PIF (India) } \\
\text { IBI (India) }\end{array}$ & $\begin{array}{l}=6.630 \\
=1.940 \\
=4.260\end{array}$ \\
\hline
\end{tabular}
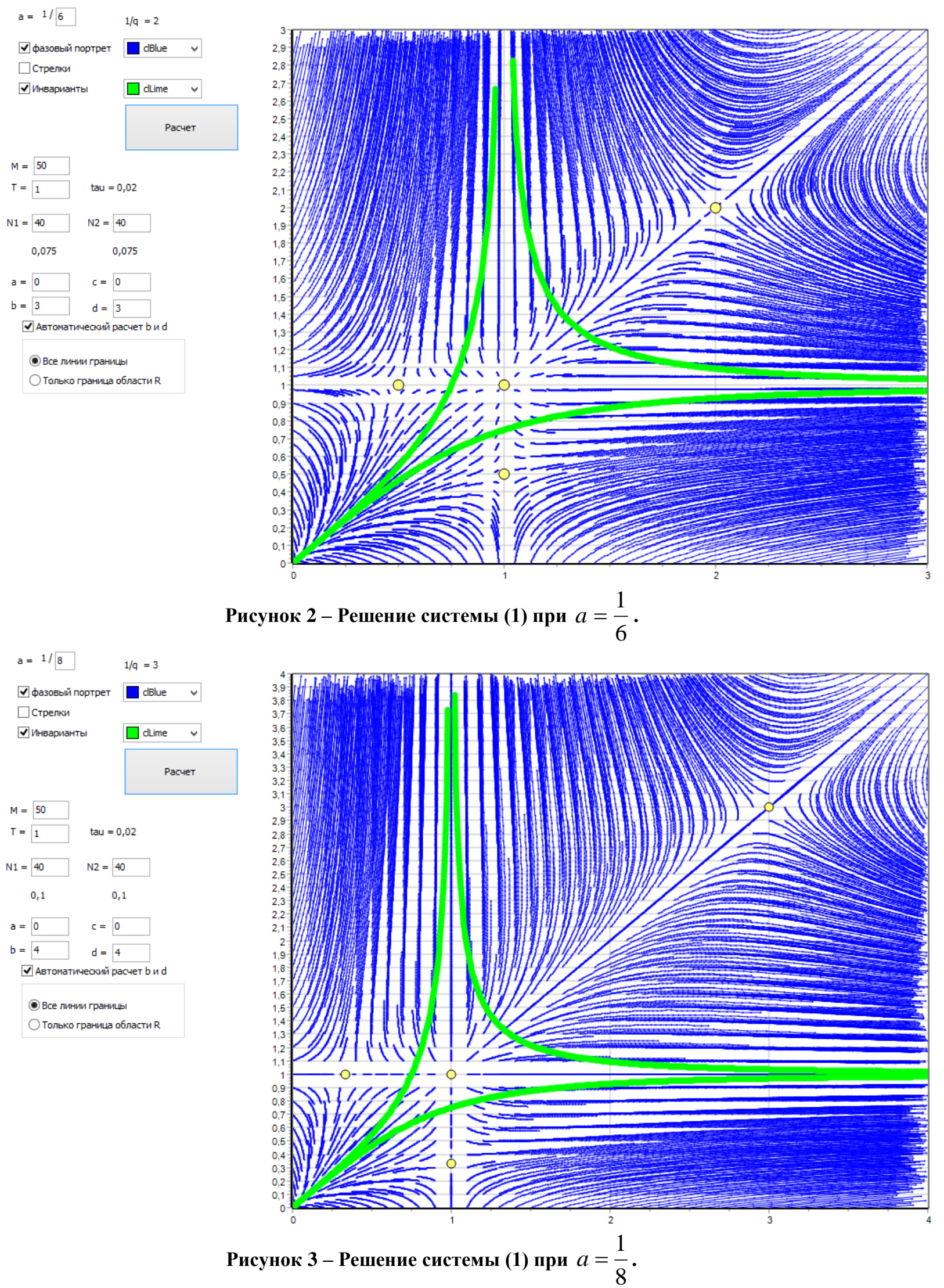

ISPC Computer technologies in science, 


\begin{tabular}{l|lr|ll|ll} 
& ISRA (India) & $=\mathbf{1 . 3 4 4}$ & SIS (USA) & $=\mathbf{0 . 9 1 2}$ & ICV (Poland) & $=\mathbf{6 . 6 3 0}$ \\
Impact Factor: & ISI (Dubai, UAE) $=\mathbf{0 . 8 2 9}$ & PUHL (Russia) $=\mathbf{0 . 2 3 4}$ & PIF (India) & $=\mathbf{1 . 9 4 0}$ \\
& GIF (Australia) & $\mathbf{0 . 5 6 4}$ & ESJI (KZ) & $=\mathbf{1 . 0 4 2}$ & IBI (India) & $\mathbf{4 . 2 6 0}$ \\
& JIF & $=\mathbf{1 . 5 0 0}$ & SJIF (Morocco) & $=\mathbf{2 . 0 3 1}$ & & \\
\hline
\end{tabular}
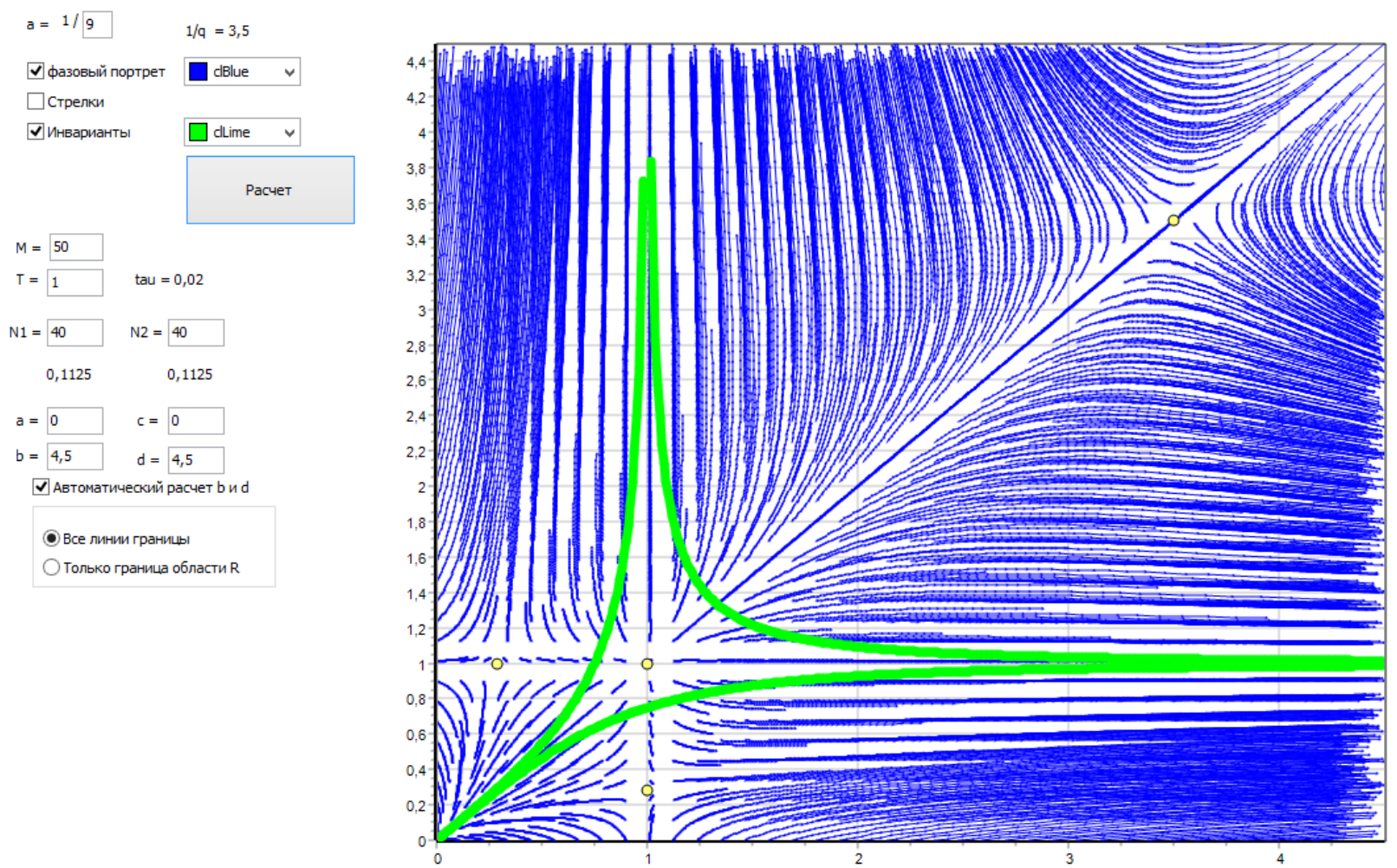

Рисунок 4 - Решение системы (1) при $a=\frac{1}{9}$.
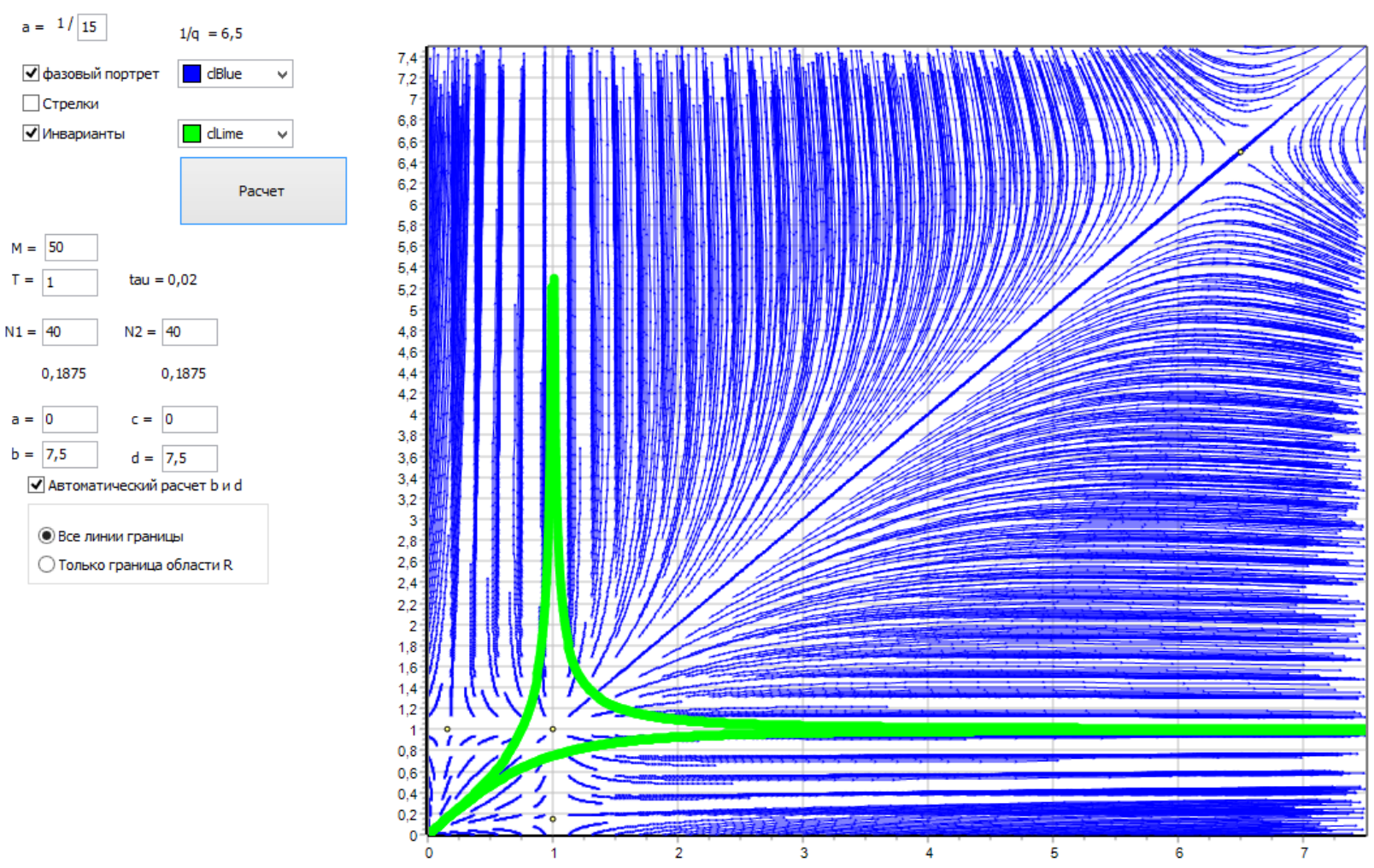

Рисунок 5 - Решение системы (1) при $a=\frac{1}{15}$. 


\begin{tabular}{l|lr|ll|ll} 
& ISRA (India) & $=\mathbf{1 . 3 4 4}$ & SIS (USA) & $=\mathbf{0 . 9 1 2}$ & ICV (Poland) & $=\mathbf{6 . 6 3 0}$ \\
Impact Factor: & ISI (Dubai, UAE) $=\mathbf{0 . 8 2 9}$ & PUHЦ (Russia) $=\mathbf{0 . 2 3 4}$ & PIF (India) & $=\mathbf{1 . 9 4 0}$ \\
& GIF (Australia) & $\mathbf{0 . 5 6 4}$ & ESJI (KZ) & $=\mathbf{1 . 0 4 2}$ & IBI (India) & $=\mathbf{4 . 2 6 0}$ \\
& JIF & $=\mathbf{1 . 5 0 0}$ & SJIF (Morocco) & $\mathbf{2 . 0 3 1}$ & & \\
\hline
\end{tabular}

Заключение.

Как мы увидели из численных экспериментов, траектории системы (1) действительно покидают область D. То есть, эксперимент полностью подтверждает теоретические выводы статьи [1].

Работа выполнена при поддержке гранта 1452/ГФ4 Министерства образования и науки Республики Казахстан на 2015-2017 годы.

\section{References:}

1. Abiev NA, Nikonorov YG (2016) The evolution of positively curved invariant Riemannian metrics on the Wallach spaces under the Ricci flow // Annals of Global Analysis and Geometry. 2016. V.50, No 1. pp.65-84.

2. Abiev NA, Arvanitoyeorgos A, Nikonorov YG, Siasos P (2014) The dynamics of the Ricci flow on generalized Wallach spaces // Differential Geometry and its Applications. 2014. V.35.pp.26-43.

3. Abiev NA, Arvanitoyeorgos A, Nikonorov YG, Siasos P (2014) The Ricci flow on some generalized Wallach spaces // Geometry and its Applications. Springer Proceedings in Mathematics and Statistics/ Eds. V.Rovenski and P.Walczak. Switzerland: Springer, 2014. V.72. pp.3-37.

4. Abiev NA (2016) Ob evolyutsii invariantnykh rimanovykh metrik na odnom klasse obobshchennykh prostranstv Uollakha pod vliyaniem normalizovannogo potoka Richchi // Matematicheskie trudy. 2016 (v pechati).
5. Lomshakov AM, Nikonorov YG, Firsov EV (2003) Invariantnye metriki Eynshteyna na trilokal'no-simmetricheskikh prostranstvakh // Matematicheskie trudy. -2003. -T.6. -№2.pp. 80-101.

6. Nikonorov YG (2000) Ob odnom klasse odnorodnykh kompaktnykh mnogoobraziy Eynshteyna // Cib.matem.zhurnal.-2000. -T.41. -№1.-pp.200-205.

7. Nikonorov YG, Rodionov ED, Slavskii VV (2007) Geometry of homogeneous Riemannian manifolds // Journal of Mathematical Sciences (New York). -2007. -V.146. -No.7. -pp.63136390.

8. Dumortier F, Llibre J, Artes J (2006) Qualitative theory of planar Differential systems. Universitext. Springer-Verlag, Berlin: 2006. xvi +298 p.

9. Samarskiy AA, Gulin AV (1989) Chislennye metody. Moscow: Nauka, GRFML,1989.-432p.

10. Bakhvalov NS (1987) Chislennye metody. Moscow: Nauka, GRFML,1987.-600 p. 